Pacific Journal of Mathematics

STRUCTURE OF RIGHT SUBDIRECTLY IRREDUCIBLE 


\title{
STRUCTURE OF RIGHT SUBDIRECTLY IRREDUCIBLE RINGS II
}

\author{
M. G. DEShPANDE
}

The object of this paper is to determine the structure and properties of right subdirectly irreducible rings which are either local or self-injective. The rings in the latter class form a special case of the so-called right $P F$ rings. By employing the notion of Feller's $X$-rings, it is proved that right $P F \quad X$-rings are finite direct sums of full matrix rings over self-injective right subdirectly irreducible rings. Thus, whether or not right $P F X$-rings are left $P F$ depends on the answer to the same question for the more elementary case of self-injective right subdirectly irreducible rings.

For a discussion of artinian and noetherian RSI rings, see [2].

1. Notation and preliminaries. All rings considered have an identity and all modules are unitary. A module $M_{R}$ is $R$-subdirectly irreducible if the intersection of all nonzero submodules of $M$ is nonzero, which will then be called the heart of $M_{R}$. A ring $R$ is $R S I$ (right subdirectly irreducible) if $R_{R}$ is $R$-subdirectly irreducible. The heart $H$ of a $R S I$ ring $R$ is a two sided ideal. These and some of the following definitions and observations are given in [2] and we rewrite them for completeness. We will always use the following notation is connection with a $R S I$ ring $R . H=$ heart, $N=H^{l}=$ $\{x \in R: x H=0\}, D=\operatorname{Hom}_{R}\left(H_{R}, H_{R}\right), \hat{R}=$ injective hull of $R_{R}, K=$ $\operatorname{Hom}_{R}(\hat{R}, \hat{R})$ and $L=\{f \in K: \operatorname{ker} f \neq 0\}$. In addition, for a local ring $R, J$ will always denote the unique maximal right ideal. A ring $R$ will be termed self-injective if $R_{R}$ is injective.

We state the following theorem showing the relationship between $R, N, H, D, K, L$ which has been proved in [2, p. 319].

THEOREM 1.1. If $R$ is $R S I$, then $R / N$ is isomorphic to a subring of the division ring $D$ and $D \cong K / L$.

In connection with $Q F-1$ algebras, faithful indecomposable modules play an important role. In the following proposition we prove that a $R S I$ ring has a unique faithful indecomposable injective module. In this respect, it may be remarked that an artinian semisimple ring which is not simple is an example of a ring for which faithful indecomposable injectives don't exist; while over the ring of integers, for each prime $p$, by using [12, p. 145, Th. 7] or otherwise, 
one can verify that $Z_{p^{\infty}}$ is a faithful indecomposable injective module, and obviously they are all nonisomorphic.

Proposition 1.2. A RSI ring $R$ has, up to isomorphism, a unique faithful indecomposable injective module.

Proof. $\quad \hat{R}_{R}$ is certainly faithful and injective. It is also indecomposable because every nonzero submodule contains $H$. Let $M_{R}$ be any other such module. If $h \in H$ is a nonzero element, then $M h \neq 0$ because $M$ is faithful. Thus, for some $m \in M, m h \neq 0$. The mapping $x \rightarrow m x$ is then an isomorphism on $H$ to $m H$ which can be extended to an isomorphism of $\hat{R}$ into $M$. If $N$ be the image of $\hat{R}$ under this isomorphism, $N$ is injective and hence a direct summand of $M$. By indecomposability of $M$, we have $N=M$ and therefore $\hat{R} \cong M$.

2. Local $R S I$ rings. We recall that a ring $R$ is a left $S$-ring in the sense of F. Kasch [5, p. 455] if each proper right ideal has a nonzero left annihilator. It is known that [11, p. 412, Th. 2.9] a (right) self-injective ring is local iff it is right uniform. In the following an analogue of this is considered for $R S I$ rings.

Proposition 2.1. A self-injective ring $R$ is $R S I$ iff it is a local left S-ring.

Proof. If $R$ is self-injective and $R S I$, then it is right uniform and thus local by the above. If $h \in H, h \neq 0$; then $h^{r}$ is a maximal right ideal and so must be the unique maximal right ideal $J$. Thus $J^{l} \neq 0$. If $A$ is any proper right ideal, then $A \subseteq J$ implies that $0 \neq J^{l} \leqq A^{l}$ and hence $R$ is a left $S$-ring. Conversely, $R$ is self-injective and local implies that it is right uniform. Since $R$ is a left $S$-ring, by [6, p. 237, 2.1] $R$ contains a copy of the simple $R$-module $R / J$. Clearly, a right uniform ring containing a minimal right ideal must be $R S I$.

The above proof shows that a $R S I$ local ring is necessarily a left S-ring. We now prove the following theorem ${ }^{1}$ which will imply that a $L S I$ (left subdirectly irreducible) left $S$-ring is local.

THEOREM 2.2. Let $R$ be a ring. For the three statements

(i) $R$ is local,

(ii) there exists a bimodule ${ }_{T} M_{R}$ such that $A^{l}$ in ${ }_{T} M$ is nonzero for any proper right ideal $A$ of $R$, and ${ }_{T} M$ is subdirectly irreducible,

1 The author is obliged to the referee for this version of the theorem and other helpful suggestions. 
(iii) ${ }_{K} \hat{R}$ is subdirectly irreducible and $R$ is a left S-ring; we have (iii) $\Rightarrow$ (ii) $\Rightarrow$ (i). If, further $R$ is $R S I$, then we also have (i) $\Rightarrow$ (iii).

Proof. (iii) $\Rightarrow$ (ii) is obvious.

(ii) $\Rightarrow$ (i). If $M_{0}$ is the heart of ${ }_{r} M$, then $M_{0} \subseteq A^{l}$ for any proper right ideal $A$ of $R$. Thus $A \subseteq A^{l r} \cong M_{0}^{r}$ which proves that $R$ is local with $J=M_{0}^{r}$.

Now we assume that $R$ is $R S I$ and prove (i) $\Rightarrow$ (iii). That $R$ is then a left $S$-ring is already noted above. Now, let $h$ and $a$ be any two nonzero elements respectively from $H$ and $\hat{R}$. Since $R$ is local and $h^{r}=J$, we have $a^{r} \cong h^{r}$. Thus $a x \rightarrow h x$ is a homomorphism of $a R$ into $H$ which can be extended to some element $f$ of $K$. Then $h=f(\alpha) \in K a$ which shows that each nonzero submodule of ${ }_{K} \hat{R}$ contains $h$ and so ${ }_{K} \hat{R}$ is subdirectly irreducible. In fact, it can be easily seen that ${ }_{K} \hat{R}$ and $R_{R}$ have the same heart $H$.

Since a $L S I$, left $S$-ring $R$ satisfies condition (ii) of the above theorem, we have in particular,

COROLLARY 2.3. For a RSI and LSI ring, the following are equivalent.

(i) $R$ is local

(ii) $R$ is a left (right) S-ring.

3. Self-injective $R S I$ rings. In a local $R S I$ ring $R$, the left annihilator $N$ of $H$, and the right annihilator $J$ of $H$ (which will be the unique maximal right ideal) need not coincide, though obviously we must have $N \cong J$. We show by an example ${ }^{2}$ that this inclusion can be proper and then prove that for self-injective rings, $N=J$.

EXAMPLE 3.1. Let $F=k\left(x_{1}, \cdots, x_{n}, \cdots\right)$ be the field of rational functions in $x_{1}, x_{2}, \cdots$ over the field $k$ of real numbers and $L$ the subring of fractions with denominators prime to $x_{1}$. Let $\alpha$ and $\beta$ denote an epimorphism and a monomorphism respectively on $L$ to $F$ given by $f\left(x_{1}, \cdots, x_{n}\right)^{\alpha}=f\left(0, x_{1}, \cdots, x_{n-1}\right)$ and $\beta=$ inclusion. Let $R$ be the ring defined by $(R,+)=L \oplus F$ and $(a, b)(c, d)=\left(a c, b c^{\alpha}+a^{\beta} d\right)$. If $h$ denotes the element $(0,1)$ of $R$, then every element of $R$ can be written as $a+h b$ and $h^{2}=0$. It can be verified that $R$ is $R S I$ with heart $H=h R$ and that $a+h b$ is a unit in $R$ iff $a$ is a unit in $L$. Since $L$ is a local ring, so is $R$. For this ring $R, N=H^{l}=\{(0, b)$ : $b \in F\}$ and $J=\left\{(a, b): a^{\alpha}=0\right\}$. Thus $N \varsubsetneqq J$.

${ }^{2}$ Professor P. M. Cohn has kindly communicated this example to the author. 
Proposition 3.2. If $R$ is self-injective and $R S I, R / N \cong D$.

Proof. It was shown in [2] that $f: R / N \rightarrow D$ defined by $f(a+N)=$ $f_{a}$, where $f_{a}: H \rightarrow H$ is the left multiplication by $a$, is a monomorphism. If $R$ is self-injective and $d: H \rightarrow H$ is any element of $D$, then there exists an element $a \in R$ such that $d=f_{a}=f(a+N)$ and $R / N \cong D$.

Corollary 3.3. If $R$ is self-injective and $R S I, N=J$.

Proof. Self-injective and $R S I$ implies local. Since $R / N$ is a division ring, $N$ must be a maximal right ideal and hence $N=J$.

We now give a characterization of $R S I$ rings in the class of self-injective rings which is analogous to McCoy's Theorem [9, p. 382, Th. 1] for the commutative subdirectly irreducible rings.

THEOREM 3.4. Let $R$ be self-injective. Then $R$ is $R S I$ iff there exists a nonzero principal right ideal $X=x R$ and an ideal $Y$ in $R$ such that

(i) $Y^{l}=X$, so that $X$ is a two sided ideal,

(ii) $X^{l}=Y$,

(iii) $R / Y$ is a division ring, and

(iv) If $a$ is an element of $Y$ not in $X$, there exists an element $b$ of $Y$ not in $X$, such that $a b=x$.

Proof. $\quad(\Rightarrow)$. If $R$ is $R S I$, we choose $X$ to be $H=h R$ and $Y=N$. Then (ii) holds by definition of $N$ and (iii) is a consequence of 3.2 above. Since $R$ is in this case local with $N=$ unique maximal right ideal, $a N=0$ if and only if $a R$ is a minimal right ideal. Thus $N^{l}=$ $H$ which proves (i). Now let $a \in N$ such that $a \notin N$. Since $H \subseteq a R$ we have $h=a b$ for some $b \in R$. (ii) implies that $b \notin H$. Also if $b \notin N$ then $b$ is a unit which implies $a=h b^{-1} \in H$ contradicting the hypothesis on $a$.

( $\Longleftarrow$. By assuming (i), (ii), (iii), and (iv) we will show that every nonzero right ideal of $R$ contains the fixed element $x$ of $R$. Accordingly, let $a$ be a nonzero element in a right ideal $A$ of $R$. If $a \notin Y$, by (iii) we have $1-a y \in Y$ for a suitable $y \in R$. Then by (ii), $(1-a y) x=0$ which implies $x=a y x \in a R \subseteq A$. If $a \in Y$ and $a \notin X$ then by (iv) we have $x \in a R \subseteq A$. Lastly, if $a \in X$, then $a=x c$ for some $c \in R$. $\quad c$ cannot be in $Y$ because then we would have $a=x c \in X Y=0$. Thus, again by (iii) $1-c d=u \in Y$ for some $d \in R$. Then $a d=x c d=$ $x(1-u)=x$ which proves that $x \in A$. Thus $R$ is $R S I$ with heart $X=x R$.

4. Right $P F \quad X$-rings. Utumi $[14$, p. 56] defined a ring $R$ to be right $P F$ if every faithful right $R$-module is completely faithful. 
These rings afford a nice generalization of $Q F$ rings and have been discussed by Azumaya [1], Osofsky [10], Kato [6, 7], Utumi [13, 14] and others. A ring $R$ is right $P F$ if and only if $[1, \mathrm{p} .701, \mathrm{Th}$. 6] it is a finite direct sum of indecomposable injective right ideals each of which contains a unique minimal right ideal. It is not known whether a right $P F$ ring is also left $P F$. Clearly, a self-injective $R S I$ ring is right $P F$. Either by using the theory of right $P F$ rings developed by the authors mentioned above, or directly as a consequence of our Theorem 2.2 it can be seen that a self-injective $R S I$ ring is left subdirectly irreducible. (In this case, $R$ is local, $\hat{R}=R$ and $K \cong R)$. We state this as a

Proposition 4.1. A self-injective RSI ring is LSI.

The following definition is due to Feller [3, p. 20, 2.2].

DEFINITION 4.2. A ring $R$ is called an $X$-ring if for every pair $e, f$ of primitive idempotents such that $e R \not f R ; a \in e R$ and $a^{r} \cap f R \neq$ 0 implies afR $=0$.

We state the following useful lemma whose proof is straightforward.

LemmA 4.3. If $A$ and $B$ are rings and $R=A \oplus B$ is the ring theoretic direct sum, then $R$ is a self-injective ring iff each of $A$ and $B$ are self-injective rings.

We are now in a position to prove the following

THEOREM 4.4. A ring $R$ is a right $P F$ X-ring if and only if $R$ is isomorphic to a finite direct sum of full matrix rings over selfinjective RSI rings. Further, this decomposition is unique.

Proof. If $S$ is a self-injective $R S I$ ring with heart $H$, then the $n \times n$ matrix ring $S_{n}$ is self-injective [13, p. 172, Th. 8.3] and is the direct sum of indecomposable right ideals $e_{i i} S_{n}, i=1,2, \cdots, n$ each of which contains a unique minimal right ideal $e_{i i} H_{n}$. Consequently $S_{n}$ is a right $P F$ ring which is trivially an $X$-ring. Now, by using 4.3 we can see that any finite direct sum of such rings is again right $P F$. In order to show that it is also an $X$-ring, it is enough to remark that $e R$ and $f R$ are nonisomorphic only if they belong to different matrix rings, in which case $e R f R=0$.

Conversely, Let $R$ be a right $P F X$-ring. Then

$$
R=e_{1} R \oplus \cdots \oplus e_{n} R
$$


where $e_{1}, \cdots, e_{n}$ are primitive and let us assume that $e_{1} R, \cdots, e_{k} R$ $(k \leqq n)$ denote a complete set of nonisomorphic right ideals among the $n$ summands in 4.5. Since each $e_{i} R$ is indecomposable and injective, it is right uniform. By the same argument as in [3, p. 20, Th. 2.3] we conclude that $R=A_{1} \oplus \cdots \oplus A_{k}$ where each $A_{i}$ is the sum of all summands in 4.5 which are isomorphic to $e_{i} R$ and $A_{1}, \cdots$, $A_{k}$ are all two sided ideals. Further, each $A_{i}$ is isomorphic to a full matrix ring over $e_{i} R e_{i}$. That this decomposition is unique follows from [4, p. 42, Th. 1]. Also, by 4.3 , each of the rings $A_{1}, \cdots, A_{k}$ is selfinjective and hence by $\left[13\right.$, p. 172 , Th. 8.3] so are the rings $e_{i} R e_{i}$. Finally, if $H_{i}$ is the unique minimal right ideal of $R$ contained in $e_{i} R$, it can be verified that $e_{i} R e_{i}$ is $R S I$ with heart $e_{i} H_{i} e_{i}$. This prove the Theorem.

As a consequence of this theorem, a right $P F X$-ring will be left $P F$ (if and) only if the self-injective $R S I$ rings over which matrix rings appear in the above decomposition are also left self-injective. The author does not know if such is always the case.

\section{REFERENCES}

1. Goro Azumaya, Completely faithful modules and selfinjective rings, Nagoya Math. J., 27, (1966), 697-708.

2. M. G. Deshpande, Structure of right subdirectly irreducible ring I, J. Alg., 17 (1971), 317-325.

3. E. H. Feller, A type of quasi-Frobenius ring, Canad. Math. Bull., 10 (1967), 19-27.

4. N. Jacobson, Structure of rings, Amer. Math. Soc. Colloq. publ. 37 (1964).

5. F. Kasch, Grundlagen Einer Theorie der Frobeniuserweiterungen, Math. Ann., 127 (1954), 453-474.

6. T. Kato, Torsionless modules, Tohoku Math. J., 20 (1968), 234-243.

7. - Some generalisations of QF-Rings, Proc. Japan Acad., 44 (1968), 114-119.

8. J. Lambek, Lectures on rings and modules, Blaisdell, Waltham, Mass. (1966).

9. N. H. McCoy, Subdirectly irreducible commutative rings, Duke Math. J., 12 (1945), 381-387.

10. B. L. Osofsky, A generalization of quasi-Frobenius rings, J. Alg., 4 (1966), 373-387.

11. M. Satyanarayana, Characterisation of local rings, Tohoku Math. J., 19 (1967), 411-416.

12. Chi Te Tsai, Report on Injective modules, Queen's papers in pure and appl. Math., No. 6, Queen's Univ., Kingston, Ontario, (1966).

13. Yuzo Utumi, On continuous rings and self-injective rings, Trans. Amer. Math. Soc., 118 (1965), 158-173.

14. —, Self-injective rings, J. Alg., 6 (1967), 56-64.

Received January 12, 1971 and in revised form June 16, 1971. A portion of this paper is included in the author's doctoral dissertation under the supervision of Professor Edmund H. Feller, submitted to University of Wisconsin-Milwaukee, in June, 1969. 


\section{PACIFIC JOURNAL OF MATHEMATICS}

\section{EDITORS}

H. SAMELSON

Stanford University

Stanford, California 94305

C. R. HobBY

University of Washington

Seattle, Washington 98105
J. DUGUNDJI

Department of Mathematics

University of Southern California

Los Angeles, California 90007

RICHARD ARENS

University of California

Los Angeles, California 90024

\section{ASSOCIATE EDITORS}

E. F. BECKENBACH

B. H. NeumanN

F WoLF

K. YoshidA

\section{SUPPORTING INSTITUTIONS}

UNIVERSITY OF BRITISH COLUMBIA

UNIVERSITY OF SOUTHERN CALIFORNIA

CALIFORNIA INSTITUTE OF TECHNOLOGY

UNIVERSITY OF CALIFORNIA

MONTANA STATE UNIVERSITY

STANFORD UNIVERSITY

UNIVERSITY OF NEVADA

NEW MEXICO STATE UNIVERSITY

OREGON STATE UNIVERSITY

UNIVERSITY OF OREGON

OSAKA UNIVERSITY

UNIVERSITY OF TOKYO

UNIVERSITY OF UTAH

WASHINGTON STATE UNIVERSITY

UNIVERSITY OF WASHINGTON

$\stackrel{*}{*} \stackrel{*}{*} \stackrel{*}{*}{ }^{*}{ }^{*}$ MMEICAN MATHEMATICAL SOCIETY

NAVAL WEAPONS CENTER

Printed in Japan by International Academic Printing Co., Ltd., Tokyo, Japan 


\section{Pacific Journal of Mathematics}

\section{Vol. 42, No. $1 \quad$ January, 1972}

Tage Bai Andersen, On Banach space valued extensions from split faces ........

David Marion Arnold, A duality for quotient divisible abelian groups of finite

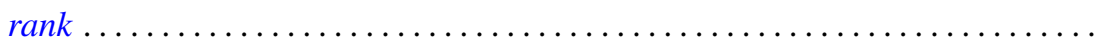

Donald Pollard Ballou, Shock sets for first order nonlinear hyperbolic

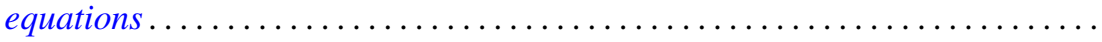

Leon Brown and Lowell J. Hansen, On the range sets of $H^{p}$ functions .........

Alexander Munro Davie and Arne Stray, Interpolation sets for analytic

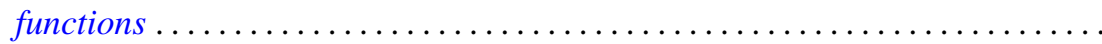

M. G. Deshpande, Structure of right subdirectly irreducible rings. II . . . . . . . . .

Barry J. Gardner, Some closure properties for torsion classes of abelian

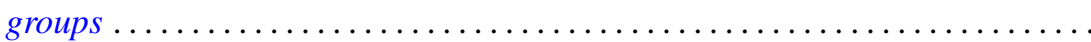

Paul Daniel Hill, Primary groups whose subgroups of smaller cardinality are

direct sums of cyclic groups . . . . . . . . . . . . . . . . . . .

Richard Allan Holzsager, When certain natural maps are equivalences .........

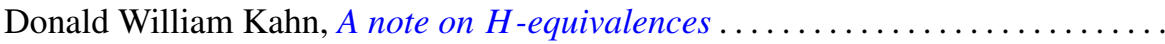

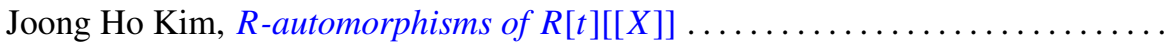

Shin'ichi Kinoshita, On elementary ideals of polyhedra in the 3-sphere.........

Andrew T. Kitchen, Watts cohomology and separability...

Vadim Komkov, A technique for the detection of oscillation of second order

ordinary differential equations .

Charles Philip Lanski and Susan Montgomery, Lie structure of prime rings of characteristic 2

Andrew Lenard, Some remarks on large Toeplitz determinants . .

Kathleen B. Levitz, A characterization of general Z.P.I.-rings. II .

Donald A. Lutz, On the reduction of rank of linear differential systems

David G. Mead, Determinantal ideals, identities, and the Wronskian ...

Arunava Mukherjea, A remark on Tonelli's theorem on integration in product

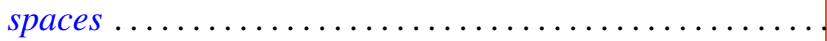

Hyo Chul Myung, A generalization of the prime radical in nonassociative rings.

John Piepenbrink, Rellich densities and an application to unconditionally nonoscillatory elliptic equations.

Michael J. Powers, Lefschetz fixed point theorems for a new class of multi-valued maps .

Aribindi Satyanarayan Rao, On the absolute matrix summability of a Fourier

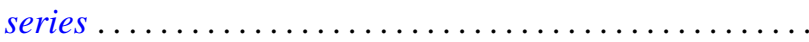

T. S. Ravisankar, On Malcev algebras ......................... 227

William Henry Ruckle, Topologies on sequences spaces . . . . . . . . . . . . . 235

Robert C. Shock, Polynomial rings over finite dimensional rings . . . . . . . . . 251

Richard Tangeman, Strong heredity in radical classes . . . . . . . . . . . . . . 259

B. R. Wenner, Finite-dimensional properties of infinite-dimensional spaces . . . . 267 